\title{
Aprovechamiento de las Redes Sociales como medio de participación ciudadana para validar posiciones e intereses de política internacional en el contexto de la diplomacia pública digital. Estudios de caso
}

\author{
The use of Social Networks as a means of citizen participation in validating \\ positions and interests of international politics in the context of digital public \\ diplomacy. Case studies
}

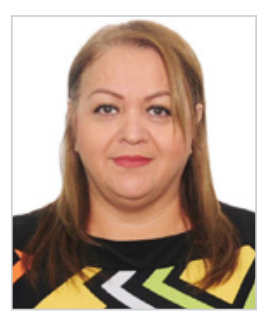
Rosmery Hernández Pereira. Doctora en Dirección Estratégica y Negocios Internacionales por la Universidad de
Sevilla. Graduada en Relaciones Internacionales por la Universidad Nacional de Costa Rica. Máster en Comercio
Internacional y Marketing por la Universidad Latinoamericana de Ciencia y Tecnología. Con especialidad en
Educación Virtual. Ha trabajado como profesora universitaria durante 27 años en las áreas de Relaciones Inter-
nacionales, Comercio Internacional y Negocios Internacionales en la Universidad Nacional de Costa Rica y la
Universidad de Costa Rica y la Universidad de Ciencia y Tecnología.. Investigadora y autora de varios artículos
en revistas y libros sobre temas relacionados con Internet, Negocios Internacionales, Diplomacia Pública y Edu-
cación Virtual. Directora de varias tesis. Consultora y Asesora Internacional de organismos gubernamentales y
empresas privadas.

Universidad Nacional, Heredia, Costa Rica

rosemery.hernandez.pereira@una.ac.cr

ORCID: 0000-0001-5125-2679

Recibido: 17/01/2019 - Aceptado: 17/05/2019

\section{Resumen}

El objetivo de la investigación es analizar el rol de las redes sociales en la diplomacia pública digital y la participación ciudadana para validar posiciones e intereses de los Estados y los gobiernos. Se fundamenta en tres fases de análisis de contenido: a) análisis interno del contenido o publicación b) causas c) efectos (Alonso, Volkens y Gómez, 2011). Durante un año (2017-2018), se dio seguimiento a las publicaciones de cuatro Estados, dos europeos y dos americanos, como estudios de caso. Se presta especial atención a las redes sociales de jefes de Estado, en relación con temas de interés internacional y se hace una comparación por medio de análisis de contenido. A partir de julio 2017 se inició una sistematización de los posts encontrados. Los principales resultados arrojaron un
Received: 17/01/2019- Accepted: 17/05/2019

\section{Abstract}

The objective of this research is to analyze the role of social networks in digital public diplomacy as well as citizen participation in validating the positions and interests of States and governments. It is based on three phases of content analysis: a) internal analysis of the content or publication b) causes c) effects (Alonso, Volkens and Gómez, 2011). For one year (2017-2018), the posts of four States, two European and two American, were followed up as case studies. Special attention was paid to the social networks of heads of state in relation to issues of international interest, and a comparison was made through content analysis. From July 2017 onward, a systemization of the posts encountered was initiated. The main results show an active, participatory role on the part

Cómo citar este artículo:

Hernández Pereira, R. (2019). Aprovechamiento de las Redes Sociales como medio de participación ciudadana para validar posiciones e intereses de política internacional en el contexto de la diplomacia pública digital. Estudios de caso. Doxa Comunicación, 28, pp. 55-77.

https://doi.org/10.31921/doxacom.n28a03 
rol activo y participativo por parte del público usuario -la ciudadanía-, puede hablarse del fenómeno del ciudadano global (Martínez, 2004). Por su parte, los decisores-gobernantes tienen un rol activo en redes sociales y buscan comunicar y validar sus acciones, casi desde el mismo momento en que se toman o se anuncia la decisión como forma de conocer las reacciones de los ciudadanos. Los Estados hacen uso de las redes sociales en el contexto de lo que se define como diplomacia pública digital.

Palabras clave:

Diplomacia pública digital; ciudadano global; Internet. of public users-citizens. We can speak of the phenomenon of the global citizen (Martínez, 2004). For their part, decision makers/ government leaders have an active role in social networks and seek to communicate and validate their actions, almost from the moment they make or announce their decisions, in order to know the reactions of citizens. States make use of social networks in the context of what is defined as digital public diplomacy.

Keywords:

Digital public diplomacy; global citizen; Internet.

\section{Introducción}

Internet puede definirse de muchas formas, desde una visión tecnológica, se concibe como la conexión entre computadoras por medio de una red, puede verse como un nuevo medio de comunicación; o puede ser abordada como un espacio donde se crean diversos tipos de relaciones (Forte, et al., 2012); todas las acepciones mencionadas pueden ser válidas. Para este trabajo, Internet se considera como un medio que favorece la participación ciudadana en temas de interés político como los relacionados con la política internacional. Desde su nacimiento a finales de los 60 y principios de los 70, Internet ha evolucionado pasando de ser una red que conecta computadoras a una red de personas que abre espacios virtuales de interacción; las limitaciones tecnológicas se han ido superando y el acceso se ha ampliado, se han expandido los servicios de banda ancha; el uso de teléfonos móviles e inteligentes se ha masificado y se ha dado una convergencia tecnológica (Internet, televisión y telefonía fija). Lo anterior "obliga a ampliar y renovar las definiciones iniciales de acceso y participación ciudadana -pensadas en un contexto mediático analógico- para actualizar su operatividad en el proceso de digitalización de señales diversas" (Linares, 2016: 39). La participación ciudadana usando Internet lleva al tema central de la participación de ciudadanos en temas de interés nacional-internacional-mundial que tradicionalmente han sido del soporte de las relaciones diplomáticas. Para enmarcar el concepto de participación ciudadana en Internet se acoge la visión de Pineda (2010), que se refiere a la comunicación que Internet facilita, entendida como: “diálogo, como espacio de negociación y mediación simbólica de las partes...que expresa en sí misma la esencia de un clima democrático que hace posible la tolerancia, la opinión, el acuerdo, pero también el disenso" (Pineda, 2010: 32-33); además, con las tecnologías de la información y la comunicación, "ese rol se ha ampliado y multiplicado ya que a través de la red Internet es posible establecer alternativas de información, comunicación y participación política más dinámicas y bilaterales” (Pineda, 2010: 36). Ello ha significado el surgimiento de una pluralidad de fuentes de poder en cuanto a la acción política de la cosa pública".

Es importante revisar datos de acceso a Internet; cada vez es más fácil conectarse, los teléfonos móviles o dispositivos inteligentes facilitan la interacción. En la última década, se ha presentado un crecimiento sostenido en la disponibilidad de las comunicaciones, especialmente gracias a la telefonía móvil celular, a la banda ancha móvil, y al desarrollo de infraestructura de banda ancha fija, lo que ha impulsado el acceso a Internet y su utilización (International Telecommunications 
Union (ITU), 2017a). En enero de 2016 había 4,2 mil millones de usuarios en el mundo (Statista, 2016: 1). Ver la figura 1, en relación con la distribución de usuarios de Internet.

Figura 1. Usuarios de Internet por región y país, 2010-2016
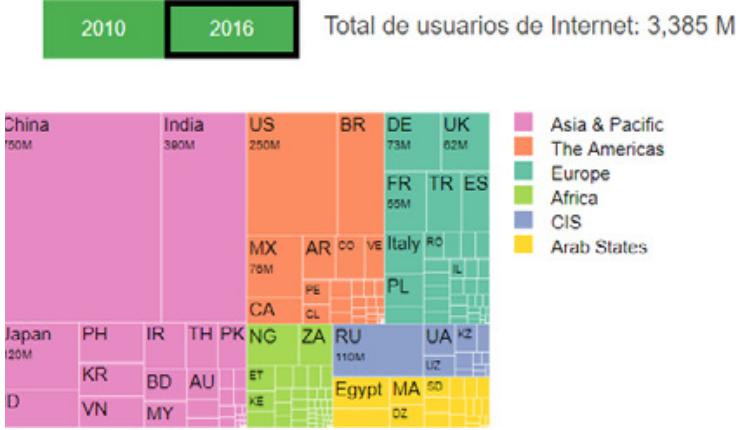

Fuente: ITU (2017b) en https://www.itu.int/en/ITU-D/Statistics/Pages/stat/treemap.aspx

Es claro que el aumento del uso de Internet incide en el uso y acceso de las redes sociales a nivel mundial; según el número de usuarios en 2018, Facebook tiene 2.300 millones de usuarios activos por mes, YouTube 1.900 millones y WhatsApp 1.500 millones, Twitter 330 millones de usuarios, Instagram 1.000 millones, LinkedIn 575 millones, (Statista, 2018a). Es claro entonces que mucha información se diseminará por estas redes y que tanto políticos como ciudadanos la están y estarán aprovechando para exponer sus criterios y manifestar sus opiniones y validar sus posiciones.

\subsection{Diplomacia pública y diplomacia pública digital}

La diplomacia pública ha hecho uso de las herramientas tradicionales para establecer contacto con el público y comunicar temas propios de la política internacional de cada Estado; estas herramientas, por su naturaleza, facilitan la información en una sola dirección que se orienta, que define la política o realiza la acción concreta hacia el público; este formato ha dificultado a los ciudadanos manifestar de forma simple y llana sus criterios respecto a las acciones de los tomadores de decisión, sobre todo en temas tan profundos como los que si discuten en el plano diplomático. Con la aparición de Internet y de la interacción que facilita la web 2.0 se abren espacios para la discusión y análisis. En forma paralela se da la construcción de un concepto al que se le denomina diplomacia pública digital, y que, en el marco de este trabajo, es necesario abordarlo para delimitar su significado.

El concepto de diplomacia pública digital no se entiende sin recurrir al concepto diplomacia pública. Algunas referencias citan el concepto de Diplomacia Pública, en el marco de la Guerra Fría; se menciona que con la creación de la Agencia de Información de Estados Unidos, en la década de los 60 del siglo XX, se dan señales de una nueva forma de hacer diplomacia valiéndose de los medios de comunicación para acercarse a los diferentes públicos (Azpíroz, 2011). Por su parte Oviamionayi (2004) señala que la diplomacia pública, como instrumento de soft power empleado por los Estados para 
promocionar la política exterior y la imagen nacional, surgió gracias a la generalización de la cultura democrática y a los avances técnicos y sociológicos de los medios de comunicación social, y a las buenas intenciones del presidente Wilson con el Open Covenants. De esta forma, la diplomacia no solo se consideraría de la forma tradicional entre gobiernos, como un instrumento propio de los Estados nacionales de corte occidental cuyos actores fundamentales son los agentes diplomáticos (Sáenz, 2016), como se concibió desde el Congreso de Viena 1815-1818, sino que se hace necesario redimensionar el concepto ya sea para ampliarlo o bien generar nuevas ramificaciones de este, al lado de la realidad que se construía en ese momento; de esta forma se consideran los nuevos espacios de las relaciones internacionales y nuevas figuras. Cuando se revisa el uso del término diplomacia pública se le atribuye el origen del concepto a Edmund Gullion. En 1965, durante el discurso de apertura del Edward R. Murrow Center of Public Diplomacy, Gullion señaló que la diplomacia pública trata la influencia en las actitudes del público en materia de política exterior más allá de la diplomacia tradicional (Cull, 2009). En este contexto se refería a la atención y el cuidado de la opinión pública en terceros países, la interacción con ciudadanos, intereses privados y grupos de interés, la difusión de información sobre las relaciones internacionales, la comunicación intensa con periodistas y profesionales de la acción exterior y la mejora de la comunicación intercultural.

Como puede notarse, el concepto ya trasciende de una diplomacia en la que los actores son solo los Estados, a una diplomacia en la que la opinión pública nacional y extranjera juegan un papel importante. Los avances en las comunicaciones pueden valorarse como un acelerador de la práctica de la diplomacia pública. En los orígenes del concepto puede verse como la relación con el público extranjero, como una forma de validar las acciones y decisiones de la política exterior de una potencia ante la opinión pública extranjera, sin embargo, su evolución ha llevado a que los gobiernos busquen la validación también de sus propios ciudadanos para sus acciones de política exterior. Según Cull (2009) los componentes de la diplomacia pública son: a) escuchar b) defensoría c) diplomacia cultural d) diplomacia de intercambio e) radiodifusión internacional.

Es importante detenerse y analizar el interés en Internet y las Redes Sociales como herramientas importantes en la práctica de la diplomacia pública, y discutir el ensanchamiento o nacimiento de un nuevo concepto, ya que nunca las tecnologías habían permitido una participación tan activa de los ciudadanos. Con la aparición de Internet entre 1968 y 1969 (Siles, 2008), las relaciones y comunicaciones entre los gobiernos, así como entre estos y las sociedades y todo el resto de las actividades que se enmarcan en relaciones sociales, dan un giro significativo. Los primeros cambios se ajustan a lo que esta tecnología permitía en sus inicios, la capacidad de transferir datos. Por el nivel de desarrollo que Internet tenía, tanto en el marco de los gobiernos y en especial de la diplomacia, su uso era limitado; se mantenía en el contexto de la privacidad política o de la diplomacia tradicional con nuevas tecnologías. A finales de la década de 1980, la red se expandió en gran medida gracias a la conexión de un gran número de ordenadores, así como a un sistema de dominios DNS (Domain Name System), hasta que aparece la World Wide Web (WWW) en 1989, creada por el inglés Tim Berners-Lee y el belga Robert Cailliau, conocida como la Web 1.0 (Rubio, 2003). Entonces la información que creaban todas las organizaciones y personas no solo era almacenada y transferible, sino fácilmente consultada. Internet empieza a tener un protagonismo público, su uso se empieza a generalizar y los gobiernos empiezan a enfrentar el reto de atender a públicos más conectados y más informados. Los medios digitales han abierto de forma significativa espacios para que los públicos 
sean escuchados, pero también para que los países utilicen estos medios para defender sus acciones, igualmente se han utilizado estas tecnologías para el posicionamiento cultural e ideológico de los países.

La diplomacia pública digital como práctica aún está en desarrollo, Cull (2009) señala que puede influir la aversión al riesgo y ansiedad en torno al control de los mensajes que comparten muchos de los Ministerios de Relaciones Exteriores, y que fue al final de la década de 1990 cuando la tecnología comenzó a jugar un rol significativo en la diplomacia pública, lo que coincide con la evolución tecnológica de la web, se inicia la creación de sitios web de países, luego en 2004 a 2006, aparecen Facebook, YouTube y Twitter, y con estas redes aparecen nuevas formas de relaciones entre tomadores de decisiones políticas y el público. Cull (2009) señala al 2008 como el año clave para estas prácticas y se refiere a las acciones del diplomático David Sarangapara, cónsul para Medios de Comunicación y Asuntos Públicos de Israel en Nueva York, usando YouTube; Saranga, además fue el primer diplomático en realizar una conferencia de prensa a nivel mundial a través de Twitter en apoyo a la guerra de su país contra las fuerzas de Hamas en Gaza. También este autor señala que el subsecretario de Estado estadounidense para la Diplomacia Pública, James K. Glassman, convencía al Departamento de Estado que trabajara en lo que él llamó Diplomacia Pública 2.0. Desde esa época se ha dado un uso significativo de los medios digitales, se da un salto a un plano de información y comunicación más público, llegando a demandar mayor interacción, con un usuario-ciudadano que tiene un rol más dinámico, utilizando los medios para incluso llegar a ser propositivo en los temas de diplomacia.

Debe resaltarse que con la diplomacia pública digital no solamente se trata de influir en la opinión pública extranjera, sino que es imprescindible la capacidad de escuchar lo que la ciudadanía tenga que decir respecto de la política exterior de su país y los espacios para que la interacción se desarrolle; los tomadores de las decisiones deben ofrecer canales apropiados para la comunicación, que faciliten el diálogo y favorezca la legitimidad de las acciones en el campo diplomático. Bajo este contexto se debe estudiar la expresión digital de la diplomacia pública, primordialmente en correspondencia con la conveniencia de atender la opinión pública nacional e internacional, e influir sobre estas. Acercándose al concepto, podría decirse que la diplomacia pública digital se enfocaría en el aprovechamiento de Internet y sus aplicaciones en la comunicación de intereses de política exterior y en la interacción con el público de interés, usando medios digitales. Es importante observar que es precisamente la Internet que se desarrolla a partir de la web 2.0, 3.0; es decir, la que permite espacios de interacción, colaboración y creación de contenidos, cómo las redes sociales, algo que no era posible en Internet 1.0, la que abre el espacio para discutir sobre la nueva diplomacia pública. En la construcción del concepto de diplomacia pública digital, algunos se refieren a la diplomacia de twitter o tuitdiplomacia como la llamó Matthias Lüfkens en 2011; otros, diplomacia pública 2.0 (Terrés, 2011); para Bassante (2014) por Diplomacia pública digital se puede entender la incorporación de las redes sociales virtuales en el ejercicio diplomático como herramienta fundamental para la consecución de objetivos de política exterior. Es interesante observar cómo este concepto no se queda en el uso de las herramientas, sino que se orienta a un análisis de uso según sus fines, en este caso los objetivos de política exterior; según este autor, no debe ningún diplomático, o ningún Ministerio de Relaciones Exteriores, desentenderse del uso de estas nuevas herramientas. En un principio estas tecnologías han sido utilizadas con fines promocionales y bajo una sola dirección, inclusive hay muy poco avance en favorecer la relación con sus públicos. Es importante, como señala Bjola (2017), reconocer la inmediatez de la comunicación e interacción que permite la diplomacia digital; en este sentido, los 
que practican la diplomacia deben estar preparados para atender de forma rápida y precisa los asuntos de interés de la política exterior de sus países; sin embargo, como el mismo autor señala es posible que se cometan errores que sea necesario corregir más adelante. También cambia la forma de la comunicación y obliga a tener formas más llanas de comunicarse, para Bjola (2017) hay un proceso de adaptación que tiene tres dimensiones, a saber: a) la dimensión de capacidad digital b) el nivel en que se analiza la aceptación o compromiso y c) la fase en que se asumen estas tecnologías en el contexto de la política exterior. La dimensión de capacidad digital se refiere al acceso y uso de los dispositivos, luego está el nivel en que se analiza la aceptación o compromiso con las de normas de comunicación digital, y luego la fase en que se asumen estas tecnologías en el contexto de la política exterior. De Bjola (2017) se toman los componentes de lo que en este trabajo se está considerando como concepto más amplio de la diplomacia pública digital, ya que no se concibe solo el acceso, sino el uso y la apropiación de esta tecnología con fines de política exterior. Siguiendo a este autor, se requiere una planificación que pasa por definir estándares de forma y uso de las tecnologías y de las comunicaciones alineados a la política exterior de cada país, todo esto estaría en el marco de lo que es la diplomacia pública digital. Hasta aquí quedan esbozados los aportes al concepto de diplomacia púbica digital para este trabajo. Se trata de un concepto en construcción y plena evolución. Por lo tanto, desde un enfoque amplio se propone entender la diplomacia pública digital como el acceso, uso y la apropiación de los recursos de interacción, comunicación, colaboración y creación de contenidos que permite la web 2.0 y 3.0 por parte de los responsables de la diplomacia de los Estados, buscando acercarse a su público nacional e internacional, ofreciéndole espacios para que manifiesten sus opiniones con respecto a temas del campo diplomático.

\subsection{Ciudadano global}

Martínez (2004) señaló que se estaba gestando una especie de ciudadanos de una comunidad global en torno a problemáticas importantes de la vida humana, entre los que se refería a Derechos Humanos, Desarrollo, Discriminación, Guerra, Globalización, Medio Ambiente, temas que ya dejaban de ser objeto de preocupación exclusiva de Estados y de instituciones oficiales o medios de comunicación; en este mismo sentido señalaba el concepto de ecosistema comunicativo de Martín (1999) y apuntaba que este era dinamizado por el uso de las nuevas tecnologías de información y comunicación que beneficiaba novedosas maneras de estar juntos y nuevas sensibilidades, pero también la interconexión de conflictos y causas. Por su parte, Araya (2001) evaluaba nuevas particularidades de agrupación y activismo ciudadano que estaban sustentados en la comunicación virtual, y lo más relevante, que el análisis de la ciudadanía debe incorporar esas nuevas formas de asociatividad; este autor consideraba que los intercambios comunicativos inducidos por estas tecnologías promovían una nueva morfología de sistemas sociales. Araya (2001) puso especial atención a la transnacionalización de las redes sociales y al ejercicio de prácticas ciudadanas más allá del territorio que define el Estado; bajo este enfoque se considera que el ciudadano global es aquel que adquiere un carácter transnacional, cuya realidad no está enmarcada únicamente por lo que ocurre dentro de sus fronteras, sino que adquiere conciencia y conocimiento de nuevas realidades y que se interesa por los temas que afectan a la humanidad y el planeta en general. Internet ha favorecido que la información fluya de forma más acelerada y con menor control por parte de los gobiernos y los medios de comunicación tradicionales, la comunicación es menos vertical y se ha vuelto más horizontal. Colombo (2005) analizó la influencia de Internet en la dimensión política, especialmente en sus posibilidades para la participación ciudadana en la toma de decisiones 
públicas; según su trabajo, las tecnologías de la comunicación y la información, en especial Internet, están facilitando estas prácticas avanzando en nuevas formas de hacer política, con una información más amplia y directa y una mayor comunicación entre representantes y representados. También Castells (2000) refería cómo el plano internacional se favorecía de Internet para diseminar la información, organizarse y movilizarse en movimientos transnacionales surgidos para defender causas de la mujer, los derechos humanos, la preservación del medio ambiente y la democracia política. Es claro entonces que el ciudadano global es aquel que accede, usa y aprovecha Internet con conciencia global, y que tiene una forma particular de practicar su ciudadanía, se expresa en la red y "busca ejercer una especie de contrapoder y presionar para el logro de objetivos comunes de interés social y colectivo, que incluso sobrepasan lo político en el sentido estricto del término, abarcando preocupaciones más globales que van más allá del ejercicio del voto y de la elección de los gobernantes" (Pineda, 2010: 32). Y en el marco de lo que se analiza en este trabajo se examinan los espacios en Internet (en especial las redes sociales) que los Estados y los gobiernos usan para comunicar temas de política internacional y validar posiciones e intereses de los Estados y que dan voz al ciudadano en el marco de esta diplomacia pública digital.

\section{Metodología}

Para este trabajo se hizo análisis de contenido en tres fases, a saber: a) análisis interno del contenido o publicación b) causas c) efectos (Alonso et al., 2011). Durante un año (2017-2018), se dio seguimiento a las publicaciones que generaron jefes de Estado y ministros de asuntos exteriores o secretarios del ramo, en relación con temas de interés internacional de cuatro Estados, dos europeos y dos americanos, con atención en las redes sociales y aunque, se había considerado revisar las páginas web de las secretarias o ministerios de relaciones exteriores, el extenso trabajo que conllevó la revisión de las redes sociales, llevó a desestimar esta acción y se dejó fuera este trabajo. No se distinguieron nacionalidades ya que se consideró el concepto de ciudadano global, aunque se podía inferir claramente que las reacciones eran en general de ciudadanos nacionales. El seguimiento se hizo en excel y una vez que se identificaron las publicaciones con más reacciones se escogieron dos por país, tratando de escoger las que más reacciones registraran y se hizo un análisis de contenido. Por ser un enfoque bastante conocido, se siguió la propuesta de Lasswell (1985), a saber: ¿Quién dice qué? ¿A través de qué canal? ¿A quién? ¿Con qué efecto? Dado que este autor estaba interesado en el estudio de los medios de comunicación de masas y de la propaganda política, esta fórmula puede aplicarse al análisis de las publicaciones en las redes sociales, ya que estas se ofrecen a las masas y buscan validar objetivos político-diplomáticos. Con base en ello, se sistematizaron en una tabla los cinco elementos básicos de la comunicación: el emisor, el mensaje, el canal y el receptor y el efecto de la comunicación. Además de la cantidad de reacciones, siguiendo criterio de experto, en los casos que se presentan en este documento, se diferenciaron mensajes considerados significativos en relación con la política internacional y se buscó entender por qué se crearon los textos disponibles, qué significan y a qué público va dirigido, antecedentes y resultados (Krippendorff, 2004). En este trabajo se revisa el número de reacciones a las publicaciones, siguiendo el modelo de pasar de letras a números para conocer el ruido o impacto generado por estas. Cuando se escogieron las publicaciones para revisar se profundizó en ellas y se usó el muestreo aleatorio simple, con una confianza de $95 \%$ y un error de $10 \%$. Es importante tener claro la característica exploratoria de este estudio y que no pretende ser concluyente sobre el objeto de 
estudio, más bien busca plantearse cuestionamientos que deben ser abordados en otros trabajos, ya que se considera que el tema sobre diplomacia pública digital, y aun más el análisis de contenido de las publicaciones en redes sociales sobre temas de interés internacional, aún están en construcción. Se contabilizan todas las reacciones, pero para una mejor comprensión y análisis en este documento se escogieron reacciones con comentario.

Los Estados seleccionados para este trabajo fueron: por América, Estados Unidos y Colombia y por Europa: España y el Reino Unido. La selección obedeció a que en los cuatro países se identificaron situaciones del contexto particulares que podrían hacer que los públicos estuvieran más atentos a las publicaciones de sus gobernantes en temas de política internacional y por ende a la revisión de las redes sociales. Para el caso de Estados Unidos, el ser una potencia mundial lo coloca siempre a la mira de su público; en Colombia destacaba el tema del Acuerdo de Paz con las FARC (Las Fuerzas Armadas Revolucionarias de Colombia-Ejército del Pueblo o FARC-EP organización guerrillera insurgente y terrorista de extrema izquierda de inspiración marxista-leninista); en España, el voto de censura del presidente y la crisis de Cataluña (se proclamó el establecimiento de la República Catalana como Estado independiente y soberano, de derecho, democrático y social, el 10 de octubre de 2017); y en Reino Unido, el Brexit (abreviatura de dos palabras en inglés, Britain y exit, que significa la salida del Reino Unido de la Unión Europea), que se dio por referéndum el 23 de junio de 2016; se dio seguimiento a Jefes de Estado y aunque se revisó lo posteado por Ministros o Secretarios de Estado, estos no se incluyeron en este trabajo porque, al cierre de la investigación, no se consideró pertinente. Debe tenerse en consideración el cambio de presidente en España. También la dificultad cuando no todos los políticos tienen red social; en ese caso siempre se revisó la red social del Ministerio de Asuntos exteriores o de las oficinas presidenciales.

\section{Estudios de caso}

\subsection{Estados Unidos}

Es importante señalar que, en este primer caso, se trata de una potencia y que algunos temas van a ser objeto entonces de mayor atención mundial. Además, en este trabajo se pone atención a la red social reconocida como la que usa el presidente norteamericano para comunicar de forma directa sus acciones y decisiones. Aunque en Estados Unidos, Facebook tiene mayor penetración que Twitter, $72 \%$ versus 35\% (Statista, 2018b), esta última red es la que Trump está usando para sus publicaciones.

Como primera publicación se destaca la reunión de Donald Trump con el presidente Vladimir Putín de Rusia. Este tema generó muchos comentarios y reacciones, contabilizadas al momento del corte en 36.000. Muchos de los comentarios se referían a la supuesta intromisión de Rusia en la campaña electoral de Estados Unidos y la no conveniencia de la reunión, dados los hallazgos de la inteligencia norteamericana. Entre los comentarios surgían posiciones que se referían a que no era sensato reunirse, dadas las acusaciones del Departamento de Justicia de Estados Unidos contra doce miembros de la inteligencia rusa por lanzar una guerra cibernética contra este país durante las elecciones de 2016. Trump hizo todo lo posible por validar su acción, como se puede ver en la figura 2 y 3 , prácticamente en el mismo momento del encuentro, con una comunicación directa y casi en tiempo real. 
Figura 2. Conferencia Helsinki 1

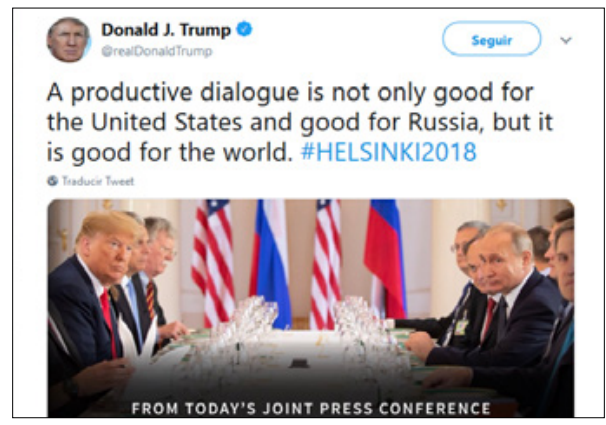

Fuente: https://twitter.com/realDonaldTrump/status/1018956970143858688

Figura 3. Conferencia de Helsinki 2

Donald J. Trump o
As I said today and many times before, "I
have GREAT confidence in MY intelligence
people." However, I also recognize that in
order to build a brighter future, we cannot
exclusively focus on the past - as the world's
two largest nuclear powers, we must get
along! \#HELSINKI2018
o Trodue rinet

Fuente: https://twitter.com/realDonaldTrump/status/1018943446583668736

Una segunda publicación analizada es la que hace el presidente Trump a partir de una conversación con el Rey Salman de Arabia Saudita, en relación con la producción de petróleo y los efectos en su precio; la figura 4 muestra el post y nuevamente este se hace casi de forma sincrónica con el momento en que se da el acontecimiento.

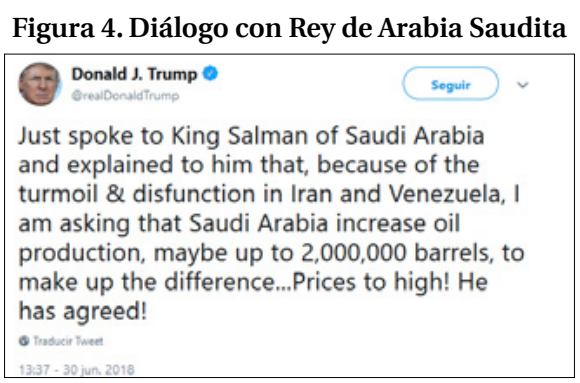

Fuente: https://twitter.com/realDonaldTrump/status/1013023608040513537 
En relación con el análisis de contenido, lo primero que hay que considerar es, a quién va dirigido el comunicado y qué efectos causa. De acuerdo al perfil de usuario de esta red, su mensaje va dirigido a personas con cierto nivel de educación, y de acuerdo con la cobertura de la red, los norteamericanos son usuarios asiduos de Twitter, aunque es claro que al estar en Internet su alcance es mundial, pero son los norteamericanos los que reaccionan muchos más al post hecho por el presidente de Estados Unidos de América, pero por ser de interés mundial, no se debe descartar que también se busca validar la acción frente a otros públicos. Es claro que las reacciones son muy diversas; en general, si el objetivo de la comunicación usando Twitter ha sido validar las acciones del presidente en materia diplomática, puede decirse que para el primer caso, las reacciones fueron mayormente negativas, de una muestra de 96 post (muestreo aleatorio simple, margen de error de $10 \%$ y confiabilidad de $95 \%$ ), el resultado fue un $30 \%$ reacciones positivas y un $70 \%$ negativas; además, aunque no se contabilizaron los datos, se observó que en general las publicaciones de quienes se decían republicanos validaron su acción. También podría pensarse que, si se buscaba atraer la atención del público hacia esa acción, el fin se logró, ya que los tuiteros reaccionaron con comentarios y memes.

Desde el rol del ciudadano global empoderado en temas de interés mundial, informado y dispuesto a opinar, vemos una realidad impensable hace unas dos décadas; sin acceso a estos recursos tecnológicos la validación o explicación de las acciones de un gobierno de forma prácticamente inmediata o sincrónica al momento de producirse no era posible.

La segunda publicación presentada aquí para estudio muestra más criterios favorables hacia la acción del presidente, un $52 \%$; podría incidir el hecho de que se trata de un tema que toca la condición económica del país y que una mejora en los precios del petróleo sería favorable para todo el país y el mundo en general. Es claro que Trump usa Twitter de manera muy particular y que ha llevado asuntos políticos antes considerados privados al dominio público, entre ellos no escapan los asuntos relativos a la diplomacia norteamericana, incluso se ha señalado que gobierna a golpe del twet; antes de asumir su mandato los medios de comunicación se referían a los tweets de Trump para identificar cuál sería la posición de gobierno en temas tan estratégicos como la relación con China y con Rusia, la doctrina nuclear, el libre comercio, la política antiterrorista, la relación con socios cercanos como Europa occidental o México. George Lakoff, profesor retirado de la Universidad de Berkeley, cataloga el uso de Twitter por parte de Trump como táctico y los enmarca en cuatro categorías: 1) Introducir una idea 2) Distraer la atención 3) Desviar o evadir la atención 4) Como globos sonda: experimenta con temas para percibir cómo se manifiesta la opinión pública. Es claro que la administración Trump comprende que hay un público que accede a las redes sociales, trata de comunicarse de forma directa, sin la interferencia de un tercero, por ejemplo un medio de comunicación o un periodista y no menosprecia la importancia de la opinión pública. Es importante señalar que el Instituto de los Caballeros de la Primera Enmienda, una organización académica de la Universidad de Columbia, presentó una demanda a nombre de siete usuarios de Twitter que habían sido bloqueados por Trump por criticarlo o mofarse de él en línea, y en junio de 2018 la jueza federal de distrito Naomi Reice Buchwald dictaminó que Trump no debería bloquear a los usuarios que le escriben en Twitter, ya que se trata de una falta a la Primera Enmienda (Constitución de los Estados Unidos) que se refiere precisamente a la libertad de expresión; el argumento fue que Twitter equivale a un foro público que el presidente no tenía el derecho de restringir. Lo anterior denota cómo se reconoce a las redes sociales cómo un nuevo espacio de comunicación e interacción y de ejercicio de ciudadanía. 


\subsection{Colombia}

El segundo caso en estudio es el gobierno de Colombia; este país reporta un importante uso de las redes sociales. Según el Ministerio de Tecnologías de la Información y la Comunicación, en los últimos años Colombia ha registrado un crecimiento exponencial en la cantidad de usuarios que se encuentran registrados en las redes sociales, Facebook y Twitter, con más de 15 millones de usuarios, Bogotá es la novena ciudad del mundo con una cifra cercana a los 6,5 millones y Colombia es uno de los países con más usuarios en redes sociales con una penetración de Internet cercana al $60 \%$, y de redes sociales de un 50\% Statista (2018). En el periodo en estudio, su mandatario también usó las redes sociales para comunicar temas de diplomacia o política internacional.

Igualmente, en este caso se presentan para el análisis, dos publicaciones, ambas se escogen con criterio de experto y por la cantidad de reacciones que provocaron. Tanto en Twitter como Facebook, el presidente Santos expuso al público colombiano la acción diplomática, pero, si se contabilizan las reacciones, hubo más en Facebook que en Twitter. En este primer caso se publica sobre la visita a los Emiratos Árabes; el presidente Santos trata de validar dos acciones, y relaciona su visita con la firma del Acuerdo de Paz de su país y trata de mostrar a sus ciudadanos que el mundo reconoce el cambio en Colombia.

Figura 5. Visita a Emiratos Árabes Unidos

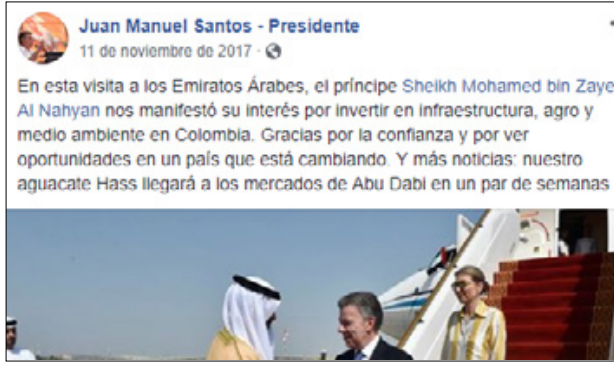

Fuente: https://www.facebook.com/330825443903/posts/10155807311843904/

Figura 6.Visita a Emiratos Árabes Unidos

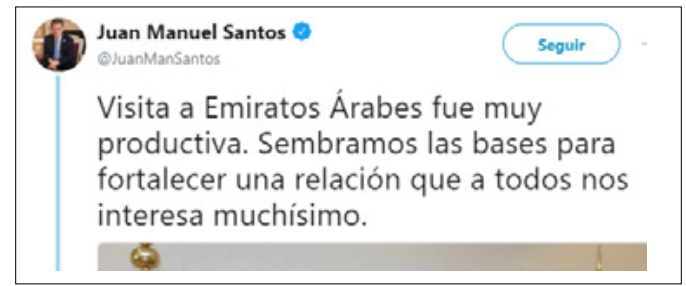

Fuente: https://twitter.com/juanmansantos/status/929704519482691584?lang=es 
La segunda publicación se da tanto en Facebook como en Twitter, se refiere a la cumbre de la Alianza del Pacífico y sus reuniones con los presidentes Vizcarra de Perú y Piñera de Chile para abordar temas de la Alianza. Es importante rescatar que hay más reacciones en Facebook que en Twitter y esto se relaciona con el número de usuarios de cada red y con el perfil de estos.

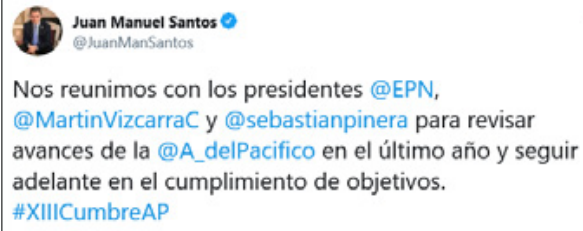

Fuente: https://twitter.com/JuanManSantos/status/1021848030075604993

Figura 8. Reunión Cumbre del Pacífico

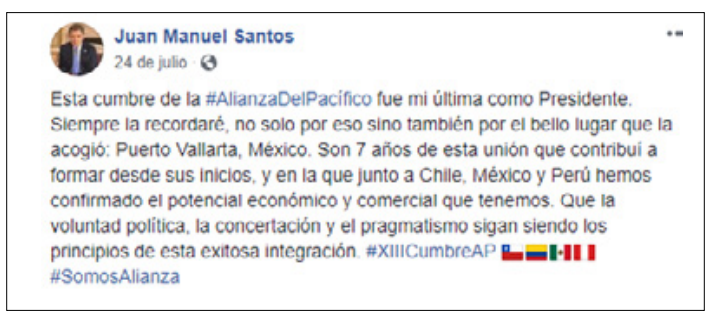

Fuente: https://www.facebook.com/330825443903/posts/10156468272748904/

En relación con la primera publicación, las reacciones fueron bastante positivas, se dieron 6.000 reacciones, con base en el muestreo (95) se obtuvo que un $16 \%$ (76 publicaciones) fueron positivas y un $24 \%$ (19) negativas. Es importante no dejar de lado los antecedentes del texto (Krippendorff, 2004) ya que, como se mencionó antes, el presidente en su mensaje buscaba recordar que Colombia estaba cambiando, rescatando así su mayor logro como mandatario.

La segunda publicación suma entre Facebook y Twitter cerca de 2.000 reacciones; el público se mostró más activo en Facebook y en general mostró un apoyo muy fuerte a la acción del presidente en este tema; el presidente hizo tres publicaciones, dos en Facebook y una en Twitter. Siguiendo el muestreo definido, se hizo una revisión de 88 reacciones, el resultado fue un $8 \%$ (7 publicaciones) mostraron oposición al presidente, sin embargo, en este caso se debe aclarar que parecía ser mayor oposición al presidente y no a la acción de la que se trataba la publicación. Por otra parte, las respuestas positivas en mucho hacían relación el logro del presidente con el proceso de paz. De los 20 post reseñados antes, 11 mencionan la palabra paz. Además, en general, los colombianos usuarios de las redes sociales que hicieron comentarios lo relacionaban con todo el mandato, ya que esta era la última cumbre a la que Santos asistiría como presidente. En general, desde el análisis de contenido, si se analiza a quién va dirigido el mensaje, puede decirse que se busca llegar a toda la po- 
blación que usa redes sociales, sin distingo de nivel de educación o clase social, ya que se usa tanto Facebook como Twitter y parece que el efecto que se desea causar es que se reconozca la labor del presidente, porque se recuerda que se está al fin del mandato, "fue mi última como Presidente". Otro elemento importante para comprender el momento histórico en que se hacen las publicaciones es que las encuestadoras estaban midiendo la popularidad del presidente saliente en Colombia y era un buen momento para recordar, en redes sociales, lo que había hecho en el marco del conflicto histórico de Colombia; el resultado parece haberse logrado, los comentarios en redes sociales les fueron positivos, con una gran asociación con el proceso de paz. Esto se relaciona con el estudio de Rodríguez et al. (2015) en el que señalaba el uso de Twitter del presidente Santos en el proceso de paz buscando generar optimismo.

\subsection{España}

El caso de España se analizó poniendo atención a que, según muchos medios, las redes sociales jugaron un papel muy importante tanto en la elección de Rajoy como presidente como en la moción de censura que sacó a Rajoy del poder; de ser así, parece razonable pensar que las redes sociales pueden jugar un rol importante en materia de diplomacia pública para España. Además, según Manfredi, Herranz y Calvo (2017) la dimensión digital es ahora parte de la estrategia de acción exterior de España, no un mero instrumento finalista y en este sentido "el modelo español de diplomacia digital busca un mayor protagonismo en la comunicación, mayor cercanía e interlocución, convencido de que la administración debe estar donde esté su ciudadanía" (Manfredi y Femenía, 2016: 20). Dado que el análisis de este trabajo se refiere a la segunda administración Rajoy, es importante reseñar que: “...la constelación de cambios digitales y audiovisuales han conducido a la necesidad de organizar una estrategia de comunicación para dar respuesta a la opinión pública internacional y a la propia demanda de los grupos españoles residentes en el exterior, alrededor de cuatro millones de personas" (Manfredi, 2017:106). Según el autor, la experiencia española es muy positiva, y las redes sociales sirven para la diseminación de información, la atención en casos de emergencia y la difusión de la agenda.

Cabe entonces analizar cómo responde la ciudadanía a la diplomacia pública digital de España. La primera publicación se refiere a la reunión de Rajoy y Trump en la oficina Oval de la Casa Blanca, figuras 8 y 9.

Figura 9. Reunión con Trump en la Casa Blanca

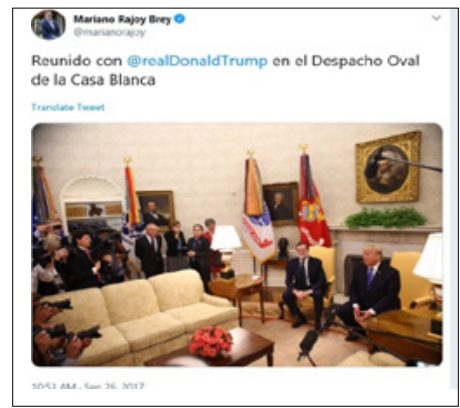

Fuente: https://twitter.com/marianorajoy/status/912721896654983168 
Aprovechamiento de las Redes Sociales como medio de participación ciudadana para validar posiciones...

Figura 10. Reunión con Trump en la Casa Blanca

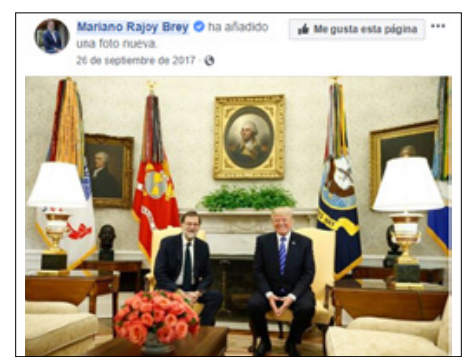

Fuente: https://www.facebook.com/54212446406/photos/a.10155722065831407/10155722066241407/?type=3

Para esta publicación se contabilizaron 4.500 reacciones; se hizo el muestreo según lo establecido en la metodología. Las reacciones a la acción que está informando Rajoy son en su mayoría negativas; a pesar de tratarse de un acercamiento a una potencia mundial, los usuarios de las redes parecen mostrar más, que un rechazo a la reunión, descontento con Rajoy; es importante recordar que en ese momento está muy presente la crisis de Cataluña que exactamente un mes después estallaría con la declaración de independencia. Incluso para ese momento, esta era considerada la peor crisis del gobierno de Rajoy; entonces puede pensarse, desde el análisis de contenido, que para el presidente español sería muy importante lograr la aceptación de sus acciones, aún más tratándose de una reunión con el presidente de una nación sumamente poderosa. Del total de las reacciones de la muestra (95), un 94\% (83) fueron de descontento y hasta de burla; no consideraban que hubiese resultados de la reunión y se criticaba al gobierno de diferentes formas, por ejemplo, por el negocio de las armas con Estados Unidos o por no atender el tema de Cataluña. Queda claro que hay un uso de la red para comunicar de forma directa el rechazo al presidente Rajoy.

Dada la situación de cambio en la presidencia de España, se consideró importante para este trabajo que la segunda publicación que se revisara fuera del nuevo presidente Sánchez. Se escogió la reunión con Macron, presidente de Francia, porque son justamente las reuniones con presidentes o jefes de gobierno las que más reacciones tuvieron en el período en que se dio seguimiento a las redes sociales, por ejemplo la reunión con Theresa May fue la tercera en reacciones en el período revisado (figuras 10 y 11 ). 
Figura 11. Primera visita de Macron a España

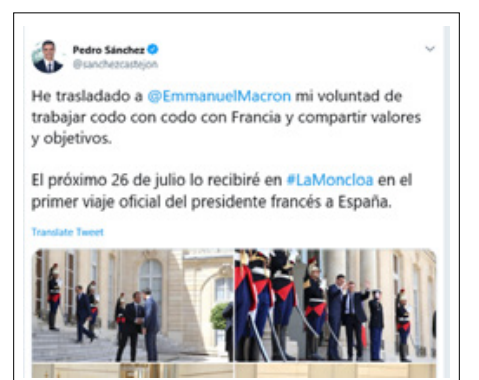

Fuente: https://twitter.com/sanchezcastejon/status/1010526521373741057/photo/1

Figura 12. Primera visita de Macron a España

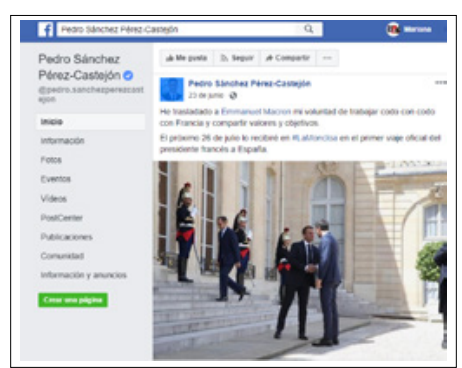

Fuente: https://www.facebook.com/750689868285972/posts/1916336015054679/

Esta es una de las primeras acciones que realiza el presidente Sánchez después de haber asumido el poder; es claro que el contexto nacional es problemático, dada la forma en que asume el poder. Otro elemento que debe tomarse como antecedente, contexto y marco de esta reunión es la situación de los migrantes en Europa, tema sumamente crítico para la UE. Puede notarse en los post y en algunos que se citan arriba, que el tema de fondo de la reunión es causante de mucho desasosiego en los usuarios de las redes sociales, y hay reacciones positivas y negativas ante el hecho de que se pueda consensuar una posición ante la crisis migratoria de Europa. En este caso, en el análisis de la muestra (91) de las 1.600 reacciones, hubo reacciones positivas al tema de un $40 \%$ (38) y un 60\% fueron negativas a la acción comunicada o bien de rechazo al presidente. Hay un elemento distintivo en este caso; se nota uniformidad en el contenido de las redes sociales del presidente Sánchez; no hace distinción según la red social, mantiene el estándar y hace una sola publicación tanto para Facebook como para Twitter, el mismo texto, la misa fotografía; pareciera haber solo interés de comunicar, sin identificar diferencias entre públicos meta. Considerando el estudio de Interactive Advertising Bureau (IAB) (2018a) las cifras de penetración de las redes sociales en España alcanzan un 85\% (25,5 millones) de la población internauta que a su vez es un 92\% (27,6 millones) de la población total española con edad entre 16 y 65 años, y un 51\% de los usuarios son mujeres, frente a un $49 \%$ de hombres, con una edad media de 38,4 años, el $74 \%$ trabaja. Se desprende de estos datos que se buscar 
Aprovechamiento de las Redes Sociales como medio de participación ciudadana para validar posiciones...

alcanzar a una población madura que trabaja, interesada en los temas de gobierno, lo que se puede validar aún más, dado que estos mismos datos indican que los españoles usan las redes sociales para conocer sobre política. Ver figura 12.

Figura 13. Actividades realizadas en Redes

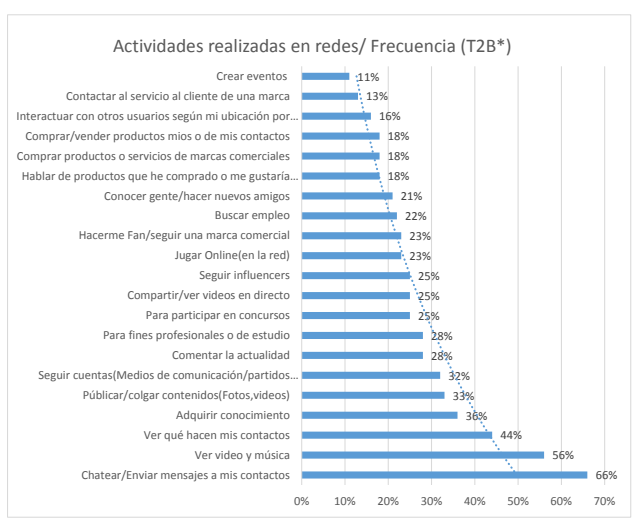

Fuente: IAB (2018b) en https://www.iab.com/insights/2017-iab-research-hub/

\subsection{Reino Unido}

La elección del Reino Unido para el estudio se hizo considerando que el Brexit puede ofrecer un contexto particular para analizar las publicaciones generadas por el gobierno. En relación con los datos de penetración de redes sociales del Reino Unido en 2017, según frecuencia de uso, el 55\% de los británicos afirmó haber accedido a algún tipo de red social todos o casi todos los días, frente al $25 \%$ de ellos que declaró no haberlo hecho nunca. Además, se estima que, en 2017, había unos 35,7 millones de usuarios de redes sociales en el Reino Unido, en comparación con los 31 millones de 2013 (Statista, 2018b). Una particularidad que resultó de la revisión fue que, en este caso, se observó mucha actividad en Twitter del Secretario de Estado para Asuntos Exteriores, Boris Jhonson, lo que abocó a darle seguimiento a estas publicaciones; sin embargo, no se incluye en este documento análisis de sus publicaciones.

El primer análisis se refiere a la cumbre en Sofía (Bulgaria) lUE-Balcanes Occidentales, del 17 de mayo de 2018. El Tweet de esta cumbre provocó más reacciones que otras publicaciones de la Primera Ministra en el período del estudio. Además se revisaron las publicaciones en Facebook en el perfil UK Prime Minister, figuras 13 y 14. 
Figura 14. Reunión con líderes de la UE y Balcanes Occidentales

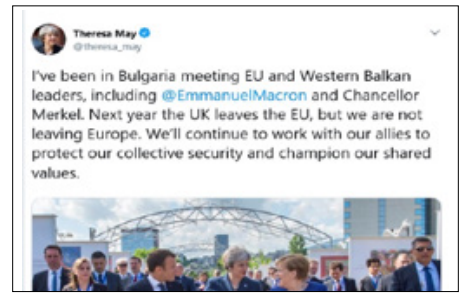

Fuente: https://twitter.com/theresa_may/status/997152059387514880

Figura 15. Momentos clave

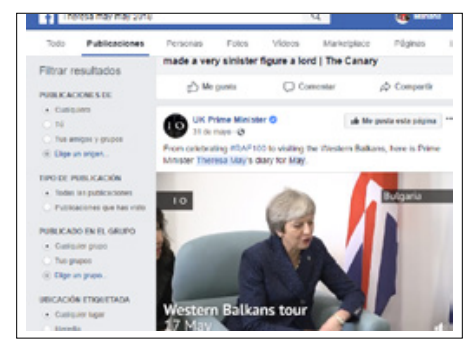

Fuente: https://www.facebook.com/10downingstreet/videos/10156520806743453/

La segunda publicación se da en el marco de la preparación de la cumbre de OTAN (Organización del Tratado del Atlántico Norte u OTAN (en inglés: North Atlantic Treaty Organization o NATO; es una alianza militar intergubernamental basada en el Tratado del Atlántico Norte o Tratado de Washington firmado el 4 de abril de 1949) del 11 y 12 de julio de 2018 . Una semana antes, la cuenta UK Prime Minister de Twitter hizo una publicación sobre una reunión entre Angela Merkel y Theresa May y los temas que abordaron como el caso de los envenenados de Amesbury y la cumbre de OTAN. Ver figura 15.

Figura 16. Theresa May y Angela Merkel hablan del Brexit y Amesbury

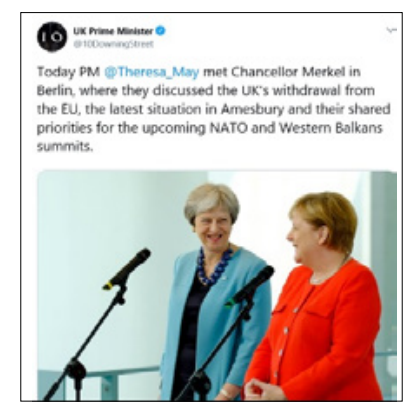

Fuente: https://twitter.com/10DowningStreet/status/1014944165417930752 
Es claro que la huella del Brexit está latente en la mente del usuario de redes sociales del Reino Unido; en las dos publicaciones que se dieron más reacciones se utiliza la palabra Brexit, por ejemplo, en 20 comentarios que se documentaron como parte de la muestra, 12 usan la palabra Brexit.

En relación con a quién va dirigido el comunicado, si se consideran los datos para 2017 (Statista; 2018b) por grupo etario, de las personas entre 16 y 24 años un 95\% usa las redes sociales, para el grupo entre 25 y 34 años un $96 \%$ usa redes sociales, para el grupo entre 34 y 44 años un $86 \%$, para las edades entre 45 y 54 años un $75 \%$ y para las edades entre 55 y 64 años un $60 \%$ usa redes sociales. Lo anterior muestra que va dirigido a una población económicamente activa y en edad de participación política, en cuanto al género, para Facebook hay mayoría de usuarias $84 \%$ que usuarios $73 \%$ y en Twitter es prácticamente el mismo porcentaje de usuarios (48\%) que usuarias (47\%), por ello puede decirse que los post buscan a ambas poblaciones sin distinción de género. En relación con los efectos que causó, puede decirse que en el caso de la primera publicación sobre la Cumbre con los líderes de UE y los Balcanes, de 90 reacciones (siguiendo el muestreo establecido) un $85 \%$ (77 reacciones) fueron negativas y un 15\% (13 reacciones fueron positivas); el tema es bastante sensible al tratar la relación Reino Unido con otros líderes de Europa en el marco del Brexit. Además, otro tema que se notó recurrente, aunque no tanto como el Brexit, fue la problemática de las migraciones. Es claro que los usuarios que dejan su comentario o reaccionan al tema tienen la impresión de que la red social da voz y que su opinión es importante. En relación con el segundo post de su reunión con Theresa May, es interesante cómo los usuarios de la red la comparan con Angela Merkel y le reclaman el liderazgo que ellos aducen tiene la Canciller Alemana en Europa; de alguna manera resalta el resentimiento por la supremacía del Reino Unido. En este caso también hay unas reacciones mayormente negativas repitiéndose casi el patrón del primer post; de una muestra de 90 reacciones, un $90 \%$ (81 reacciones) fueron negativas y un $9 \%$ (9 reacciones fueron positivas). Es importante poner atención a la preocupación de Theresa May por el efecto de las redes sociales para los políticos, diciendo que estas se están convirtiendo en espacios para la intimidación y abuso de las figuras públicas. Esto demuestra, de alguna forma, que no se siente a gusto con ellas y que no parece tener una estrategia clara de su uso y aprovechamiento. Aquí cabe señalar que, en gran Bretaña, "la diplomacia pública es una forma de comunicarse con personas en un mundo muy conectado y digitalizado. Y si bien es probablemente una de las formas más eficientes de comunicación entre los gobiernos y las personas, es al mismo tiempo una de las prácticas más difíciles" (Raskovick,2018: 63).

\section{Conclusiones}

Este estudio aporta hallazgos que permiten ir definiendo enfoques adecuados para el abordaje de la participación ciudadana en temas de la política internacional en el marco de la diplomacia pública digital y específicamente de las redes sociales. Se evidencia cómo el ciudadano global accede, usa y se apropia de los recursos de interacción, comunicación y creación de contenidos que ofrece la Internet para interactuar con los responsables de la diplomacia de los Estados; por otra parte, estos, a su vez, consideran importante comunicar y participar a sus públicos en estos temas.

En el caso de Estados Unidos, la forma en que su presidente viene utilizando las redes sociales, en especial Twitter, en temas propios de la política internacional, demuestra el gran valor que el mandatario le da como medio de comunicación 
directo con el público, y de la misma manera responden sus ciudadanos. Se deduce entonces una comunicación y participación política más dinámica y bilateral, como expresaba Cardoso (2008). Este fenómeno debe seguirse con especial atención porque al tratarse de una potencia mundial pude generar que otros gobernantes emulen la práctica, y lo mismo ocurra con ciudadanos de otros países. Desde el análisis de contenido (Krippendorff, 2004), se nota un claro aprovechamiento de las redes sociales por parte del gobierno norteamericano en informar prácticamente de forma inmediata cuando acontece la acción y no esperar que sean otros medios, es decir, un tercero, que ponga a disposición la información; es claro que los contenidos se crean para informar y generar interacción con el público sin ninguna intervención.

En el caso colombiano y del Reino Unido, se comprueba lo señalado por Cull (2009) en relación con la atención y el cuidado de la opinión pública y la interacción con ciudadanos a través de la explicación de los aspectos que forman la ideología del actor, en este caso el gobierno. En prácticamente cada publicación se hacía referencia directa o indirecta a los procesos de paz en Colombia y al Brexit del Reino Unido, los post no se recibían por parte del público de forma aislada, sino que por el contrario, las reacciones a este se relacionaban con al escenario o contexto del país o la región; por ello se considera necesario revisar en otros estudios las conexiones que hace el ciudadano entre las acciones de la política interna y exterior en un marco más amplio, porque parece denotarse que gracias a la información que se ofrece por medio de las redes sociales, el ciudadano tiene mayor claridad de la interconexión de conflictos tal como señaló Araya (2001).

Cabe destacar que, para el caso español, a pesar del cambio de administración, razón por la que se revisan post de ambas administraciones, Rajoy y Sánchez, hay una estrategia de exponer y defender los objetivos diplomáticos usando la diplomacia pública digital y en forma particular las redes sociales. Además, esta conclusión se fortalece con la literatura revisada que documenta que la dimensión digital es ahora parte de la estrategia de acción exterior de España y que el modelo español de diplomacia digital busca mayor cercanía e interlocución (Manfredi, Herranz y Calvo, 2017). Puede entonces hablarse de una estrategia de Estado más que de una práctica de un gobierno en particular.

En relación con el concepto de ciudadano global, se concluye que mantiene vigencia lo expuesto por Colombo (2005) cuando señaló que las tecnologías de la comunicación y la información están facilitando y avanzando en nuevas formas de hacer política, con una información más amplia y directa y una mayor comunicación entre representantes y representados. Y que Internet favorece la diseminación de la información y se defienden causas y que busca ejercer el contrapoder para el logro de objetivos colectivos (Castells, 1997), aunque hay que profundizar, como lo sugiere el trabajo de Baack (2015), para conocer si lo que favorecen las redes sociales es una mayor información, o una mayor participación política, o una mayor cantidad de medios para expresarse.

En relación con estudios futuros, no se puede desatender la dinámica propia del avance tecnológico de las comunicaciones que Internet seguirá favoreciendo. Es probable que los avances en la Inteligencia Artificial y Big data lleguen a hacer que los políticos y los ciudadanos puedan crear sus propios algoritmos para analizar estos temas y sugerir acciones y decisiones en materia de política internacional y que claramente afecten las decisiones que se tomen en materia diplomática, por tanto; el estudio de estos temas seguirá siendo pertinente. Sin embargo, mientras esto sucede, teniendo en cuenta lo que aduce Lidén (2016) que no se ha prestado mucha atención al lado de la oferta, a saber, la presencia en línea de los actores políticos y las estructuras creadas por ellos, queda aún mucho por estudiar. Las redes sociales, si bien son 
muy ricas en ofrecernos reacciones o acciones de los políticos y ciudadanos, se debe prestar atención a otras formas de comunicación que Internet ha creado y seguirá creando.

También se debe continuar analizando lo que expone Baack (2015), en relación con que la información es una condición para la participación política, por lo tanto, dado que las redes sociales ofrecen información de forma más abierta y accesible en temas tan densos como los de política internacional, hay que profundizar en el estudio de si estas realmente favorecen una mayor participación ciudadana en los procesos de toma de decisiones de los Estados en temas de política internacional.

Otro tema que hay que estudiar es el papel de las redes sociales como origen de información de las acciones de los gobiernos y que lleva a otros medios, como CNN, a utilizarlos como fuente primaria, así como el interés de los gobiernos de no utilizar los medios de comunicación tradicionales para llegar a su público, ya que se denota una disposición de los tomadores de decisiones para interactuar con sus ciudadanos sin ninguna tercerización, y además que mucha de la información que llega a públicos nacionales y extranjeros es generada desde redes sociales oficiales.

Cabe decir entonces que el tema en estudio no está agotado, sino que abre nuevas posibilidades de investigación que deben seguirse atendiendo.

\section{Referencias Bibliográficas}

Alonso, S., Volkens, A. y Gómez, B. (2011). Análisis de contenido de textos políticos. Un enfoque cuantitativo. Recuperado de https://www.researchgate.net/publication/257937297 [Fecha de consulta: 20 de agosto de 2018].

Araya, R. (2001). La globalización de los ciudadanos- El caso Attac. Nueva Sociedad, 6(176), 87-101. Recuperado de http:// nuso.org/revista/176/entre-la-globalizacion-y-el-multilateralismo-cooperativo/\#tema-central

Azpíroz, M. (2011). La diplomacia pública estadounidense de la "guerra contra el terror": Análisis y evaluación de su influencia en la prensa española (Tesis doctoral). Recuperado de http://dadun.unav.edu/ [Fecha de consulta: 20 de agosto de 2018].

Bassante, D. (2016). Diplomacia Digital. Las Relaciones Internacionales en tiempos de Twitter y Facebook. Revista Afese, 59 (59), 73-89. Recuperado de http://www.revistaafese.org/ojsAfese/index.php/afese/article/view/291

Baack, S. (2015). Datafication and empowerment: How the open data movement re-articulates notions of democracy, participation, and journalism. Big Data \& Society July-December 2015: 1-11. DOI: 10.1177/2053951715594634

Bassets, M. (26 de diciembre de 2016). Trump transforma a golpe de 'tuit' la política exterior de Estados Unidos. El País. Recuperado de https://elpais.com/internacional/2016/12/25/estados_unidos/1482698516_892759.html

BBC Mundo. (24 de mayo de 2018). Estados Unidos: por qué una jueza impidió a Donald Trump bloquear en Twitter a los usuarios que lo critican. Redacción BBC Mundo. Recuperado de https://www.bbc.com/mundo/ noticias-internacional-44233965 
Bjola, C. (2017). Adapting Diplomacy to the Digital Age:Managing the Organisational Culture of Ministries of Foreign Affairs (Informe de investigación No. 09) Recuperado del sitio de internet Stiftung Wissenschaft und Politik: https://www. swp-berlin.org/fileadmin/contents/products/arbeitspapiere/WP_Diplomacy21_No9_Corneliu_Bjola_01.pdf [Fecha de consulta: 20 de agosto de 2018].

Cardoso, G. (2008). Los medios de comunicación en la sociedad red [versión Portable Document Format]. Recuperado de https://dialnet.unirioja.es/descarga/articulo/2997216.pdf [Fecha de consulta: 15 de setiembre de 2018].

Castells, M. (2000). La era de la información: economía, sociedad y cultura. Volumen I: LA SOCIEDAD RED [versión Portable Document Format]. Recuperado de http://www.felsemiotica.org/site/wp-content/uploads/2014/10/LA_SOCIEDAD_RED-Castells-copia.pdf [Fecha de consulta: 15 de setiembre de 2018].

Collado, A. (2012). Campaña electoral en 140 caracteres. Cuadernos de pensamientos políticos FAES, 4(33), 203-216. Recuperado de https://dialnet.unirioja.es/servlet/articulo?codigo=3807053

Colombo, C. (2005). Participación ciudadana en la red. Recuperado de http://www.gestioncultural.org/ficheros/1_1316765188_bgc11-CColombo.pdf [Fecha de consulta: 15 de setiembre de 2018].

Cull, N. (2009). Diplomacia pública: consideraciones teóricas. Revista Mexicana de Política Exterior, 3(85), 55-92. Recuperado de https://revistadigital.sre.gob.mx/index.php/numeros-anteriores/116-rmpe85

Forte, M., Pignuoli, S., Calise, S., Palacios, M.yZitello, M. (2012). Las TIC como problema de la teoría sociológica. Entramados y Perspectivas, 2(2), 205-226. Recuperado de https://publicaciones.sociales.uba.ar/index.php/entramadosyperspectivas/ issue/viewIssue/15/24

García, A., De Ayala, M. y García, B. (2013). Hábitos de uso en Internet y en las redes sociales de los adolescentes españoles. Comunicar, 21(41), 195-204. DOI: http://dx.doi.org/10.3916/C41-2013-19

GlobalWeb Index. (7 de noviembre de 2017). La importancia de los teléfonos inteligentes sigue aumentando [Mensaje en un Blog]. Recuperado de https://blog.globalwebindex.com/chart-of-the-day/smartphone-importance-continues-increase/ IAB. (2018a). Estudio Anual de Redes Sociales 2018. Recuperado de https://www.iab.com/insights/2017-iab-research-hub/ [Fecha de consulta: 15 de setiembre de 2018].

ITU. (2017a). Informe sobre la Medición de la Sociedad de la Información de 2017. Resumen. Recuperado de https://www. itu.int/en/ITU-D/Statistics/ [Fecha de consulta: 15 de setiembre de 2018].

ITU. (2017b). Usuarios de internet por región y país, 2010-2016 [Figura]. Recuperado de https://www.itu.int/en/ITU-D/ Statistics/Pages/stat/treemap.aspx [Fecha de consulta: 20 de setiembre de 2018].

Krippendorff, K. (2004). Content Analysis. An Introduction to Its Methodology [versión Portable Document Format]. Recuperado de https://usu.instructure.com/files/67974091/download?download_frd=1\&verifier=qSU7xYWottTv6ZBbrn2n9XVCX50rZ9b7EaHVx6Uq [Fecha de consulta: 20 de setiembre de 2018]. 
Aprovechamiento de las Redes Sociales como medio de participación ciudadana para validar posiciones...

Lasswell, H. (1985). Estructura y función de la comunicación en la sociedad [versión Portable Document Format]. Recuperado de http://www.periodismo.uchile.cl/talleres/teoriacomunicacion/archivos/lasswell.pdf [Fecha de consulta: 20 de setiembre de 2018]

Liden, G. (2016) Inequality in Local Digital Politics: How Different Preconditions for Citizen Engagement Can Be Explained. sociedad [versión Portable Document Format]. Recuperado de https://onlinelibrary.wiley.com/doi/pdf/10.1002/ poi3.122. [Fecha de consulta: 23 de abril de 2019]

Linares, A. (2016). Acceso y participación ciudadana. Una actualización de indicadores para la democratización de las comunicaciones. Intercom, 39(3), 37-54. DOI: 10.1590/1809-5844201633

Manfredi, J. y Femenía, C. (2016). La diplomacia española ante el reto digital [versión Portable Document Format]. Recuperado de http://www.exteriores.gob.es/Portal/es/SalaDePrensa/Multimedia/Documents/2016_FEBRERO_LIBRO\%20 DIPLOMACIA\%20DIGITAL\%20version\%20web.pdf [Fecha de consulta: 20 de setiembre de 2018]

Manfredi, J., Herranz, J. y Calvo, L. (2017). Transparencia y diplomacia: nuevas demandas sociales y rutinas profesionales. Revista Latina de Comunicación Social, (72), 832-848. DOI: 10.4185/RLCS-2017-1195

Martín, J. (1999). La educación en el ecosistema comunicativo. Comunicar, 7(13),13-21. Recuperado de https://www.revistacomunicar.com/indice/articulo.php?numero=13-1999-03

Martínez, G. (2004). Internet y ciudadanía global: Procesos de producción de representaciones sociales de ciudadanía en tiempos de globalización. Aposta, (9), 1-20. Recuperado de http://www.apostadigital.com/revistav3/hemeroteca/martinez.pdf

Ministerio de Tecnologías de la Información y las Comunicaciones (MINTIC). (10 de junio de 2018). Colombia es uno de los países con más usuarios en redes sociales en la región. MINTIC. Recuperado de https://www.mintic.gov.co/portal/604/w3-article-2713.html

Oviamionayi, V. (2004). Diplomacia pública en la bibliografía actual. Ámbitos. Revista Internacional de Comunicación, (12), 215-236. Recuperado de http://www.redalyc.org/articulo.oa?id=16801212

Pew Research Center. (2015). Demografía de plataformas de redes sociales clave. Washington, DC., Estados Unidos: Pew Research Center. Recuperado de http://www.pewinternet.org/2015/01/09/demographics-of-key-social-networking-platforms-2/\#twitter [Fecha de consulta: 20 de agosto de 2018]

Pineda, M. (2010). Las nuevas prácticas ciudadanas en internet y las oportunidades para políticas de comunicación participativas. Revista Estudios Culturales, 2(6), 31-46. Recuperado de https://dialnet.unirioja.es/servlet/ articulo?codigo $=3739703$

Rodríguez, C., Romero, M., Navarro, L., Sáenz, A., Hernández, L. y Páez, O. (2015). Medios de Redes Sociales y Polarización en Colombia. Uso de Twitter durante el Proceso de Paz Colombiano. Recuperado de http://fnpi.org/es/etica-segura/estudio-analiza-polarizaci\%C3\%B3n-en-twitter-durante-proceso-de-paz-colombiano [Fecha de consulta: 20 de setiembre de 2018] 
Rubio, A. (2003). Historia e internet: Aproximación al futuro de la labor investigadora. Recuperado de https://webs.ucm. es/info/hcs/angel/articulos/historiaeinternet.pdf [Fecha de consulta: 20 de setiembre de 2018].

Sáenz, B. (2016). La codificación del derecho diplomático: Una perspectiva histórica. Comillas Journal of International Relations, 3(6), 61-70. DOI: cir. i06.y2016.001

Siles, I. (2008). Por un sueño en.red.ado. Una historia de Internet en Costa Rica. San José, Costa Rica: Editorial de la Universidad de Costa Rica

Statista. (2016). Número de usuarios de Internet en el mundo 2016, por región (en millones). Hamburgo, Alemania: Statista Estadísticas. Recuperado de https:// es.statista.com/estadisticas/598720/numero-de-usuarios-de-internet-por-regionesen-el-mundo/ [Fecha de consulta: 25 de setiembre de 2018].

Statista. (2018a). Ranking de las principales redes sociales a nivel mundial según el número de usuarios activos en abril de 2018 (en millones) Recuperado de https://es.statista.com/estadisticas/600712/ranking-mundial-de-redes-sociales-por-numero-de-usuarios/ [Fecha de consulta: 25 de setiembre de 2018].

Statista. (2018b). Ranking de las principales redes sociales a nivel mundial según el número de usuarios activos en abril de 2018 (en millones). Hamburgo, Alemania: Statista Estadísticas. Recuperado de https://es.statista.com/estadisticas/600712/ranking-mundial-de-redes-sociales-por-numero-de-usuarios/ [Fecha de consulta: 25 de setiembre de 2018]. Terrés, G. (2011). “Nuevas herramientas de comunicación para la Diplomacia Mexicana” (Tesis de maestría). Recuperado de http://www.septien.edu.mx/catalogo/ [Fecha de consulta: 25 de setiembre de 2018].

Vincos Blog. (2018a). Mapa mundial de redes sociales [Figura]. Recuperado de http://vincos.it/world-map-of-social-networks/ [Fecha de consulta: 25 de setiembre de 2018].

Vincos Blog. (5 de febrero de 2018b). El mapa de las redes sociales en el mundo-enero de 2018 [Mensaje en un Blog]. Recuperado de http://vincos.it/2018/02/05/la-mappa-dei-social-network-nel-mondo-gennaio-2018/

We Are Social. (30 de enero de 2018). Digital in 2018: World's internet users pass the 4 billion mark [Mensaje en un Blog]. Recuperado de https://wearesocial.com/blog/2018/01/global-digital-report-2018 
